\title{
In vivo Evaluation of Microemulsion-Based Transdermal Gel of Coumarins from Cortex Daphnes
}

\author{
Jinli Deng \\ School of science, Tianjin University, \\ Tianjin, China; \\ iloveyou-china@163.com
}

Xia Feng*

School of science, Tianjin University, Tianjin, China;

f_x@tju.edu.cn,

\section{Li Ma*}

Department of Pharmacy, Tianjin Institutes of Pharmaceutical Research,

Tianjin, China;

zhihua504@163.com

\begin{abstract}
Objetive The aim of the present study was to investigate the pharmacokinetics of coumarins from cortex daphnes (CCD) in a microemulsion (ME) gel transdermal delivery system with a newly developed HPLC method. Methods The pharmacokinetics of the microemulsion containing CCD was studied in rabbits with the established HPLC method. Results The results showed that among the three index compounds of CCD, the daphnin and daphne-8-O- $\beta$-D-glucoside from $\mathrm{ME}$ gel presented significant higher $\mathrm{AUC}_{(\mathbf{0}-\infty)}$ and $\mathrm{C}_{\max }$ compared to the conventional preparation $(\mathrm{CP})$, while daphnetin showed similar $\mathrm{AUC}_{(0-\infty)}$ and higher $\mathrm{C}_{\max }$ compared to the latter. Conclusion Hence, the established HPLC method was suitable to analyze the CCD components in ME and the ME gel could be a promising transdermal delivery system for CCD.

Keywords-coumarins; Cortex daphnes; microemulsion; pharmacokinetic; transdermal delivery
\end{abstract}

\section{INTRODUCTION}

Cortex daphnes (Zushima) are a type of traditional Chinese medicine. They have the effect of dispelling pathogenic wind, eliminating dampness, promoting blood circulation to arrest pain, and have been accepted by the public in China for a long time because of their analgesic action and widespread use in the treatment of rheumatism, arthritis and rheumatoid arthritis $[1,2]$. Chemically, extracts of cortex daphnes contain several classes of natural products including coumarins, diterpenoids, lignans, and flavonoids. Coumarins, mainly composed of three compounds, namely daphnin, daphnetin-8-O- $\beta$-D- glucoside and

\author{
Feng Han \\ Department of Pharmacy, Tianjin Institutes of \\ Pharmaceutical Research, \\ Tianjin, China; \\ hanf@tjipr.com
}

Xingcui Qu

Bo Teng Biological Pharmaceutical Technology

Co., Ltd,

Guangzhou, China.

498042475@qq.com

\author{
Yafei Wang \\ School of science, Tianjin University, \\ Tianjin 300072, China; \\ wyf77790@126.com.
}

daphnetin, have been considered as the active constituents of cortex daphnes and are usually used as the index of the drug[3].

Commonly used dosage forms of cortex daphnes are sticking plaster, tablets, ointment, pills, and spray[4,5]. However, side effects[6,7] including abdominal discomfort or nausea, pruritus, and low absorption rate were also found in clinical use. New preparations of cortex daphnes need to be developed in order to reduce adverse reactions and improve therapeutic effects. Microemulsion (ME) formulation is considered to be a promising one.

Microemulsions are isotropic, thermodynamically stable, and transparent (or translucent) systems of oil, water and surfactant [8]. MEs are considered efficient carriers in drug delivery systems and effective vehicles for solubilization of both hydrophilic and lipophilic drugs $[9,10]$.

In this study, we investigated the pharmacokinetics of microemulsion containing CCD transdermal delivery system in rabbits with a newly developed high performance liquid chromatography (HPLC) method. The aim was to develop a new formulation of cortex daphnes with high efficacy and less adverse reaction for further research and application.

\section{MATERIALS AND METHODS}

\section{A. Materials}

The coumarins, daphnin (>98\%) and daphnetin-8- 
O- $\beta$-D-glucoside (>98\%) were extracted from cortex daphnes in our laboratory with ethanol reflux method. Methanol (GR) was purchased from Tianjin Ke Kang De Ltd. (China). Phosphate was purchased from Tianjin Feng Chuan Chemical Reagent Co., Ltd (China). Ferulic acid, Heparin sodium, and daphnetin were purchased from the National Institute for the Control of Pharmaceutical and Biological Products (China).

\section{B. Preparation of $M E$ gel of $C C D$}

ME gel of CCD was lab-made that contained $384 \mathrm{mg}$ daphnin, $307 \mathrm{mg}$ daphnetin-8-O- $\beta$-D-glucoside and 209 mg daphnetin.

\section{High performance liquid chromatography (HPLC) Analysis}

\section{1) HPLC Method}

The analyses were performed with an Agilent 1100 HPLC (Aglient Technologies, America). A Prodigy ODS (3) $(4.6 \times 250 \mathrm{~mm}, 5 \mu \mathrm{m})$ column was used to separate and test the drug ingredients. The mobile phase was the mixture of methanol and $0.5 \%$ phosphonic acid $(30: 70, \mathrm{v} / \mathrm{v})$ and pumped at a flow rate of $1.0 \mathrm{~mL} / \mathrm{min}$. The effluent was monitored at $325 \mathrm{~nm}$ with ferulic acid as internal standard. The column temperature was $35^{\circ} \mathrm{C}$ and the injection volume was $10 \mu \mathrm{L}$ each time.

\section{2) Solutions}

Daphnin (22.70 mg), daphnetin-8-O- $\beta$-D-glucoside $(21.83 \mathrm{mg})$ and daphnetin $(14.38 \mathrm{mg})$ were accurately weighed and dissolved in $50 \%$ methanol to $25 \mathrm{~mL}$ to make the stock solution. Series of standard solutions were prepared by dilute the stock solutions with $50 \%$ methanol to certain concentration. The internal standard (ferulic acid) solution was prepared in $50 \%$ methanol at a concentration of $229.4 \mu \mathrm{g} / \mathrm{mL}$.

$50 \mu \mathrm{L}$ of the standard solution were added to $500 \mu \mathrm{L}$ of blank rabbit plasma to obtain the calibration standard solutions of the three index compounds. The low, medium and high concentration solutions were prepared in the same way as the quality control (QC) samples.

\section{3 ) Validation of assay method}

The selectivity of the HPLC method was investigated by comparing the chromatograms of blank plasma, calibration standard solutions of the three index compounds added with the internal standard, and blank plasma sample added CCD. The linearity of each calibration curve was determined by plotting the peak-area ratio $(y)$ of the analyte to internal standard versus the nominal concentration $(x)$ of the three index compounds. The calibration curves were obtained with weighted $(1 / \mathrm{x} 2)$ linear regression analysis.

The results obtained with QC samples in three experimental runs were used to evaluate the accuracy and precision of the method. The assay precision was expressed as the relative standard deviation (RSD). The accuracy was determined by comparing the concentration calculated from the calibration curve with the original one.
The extraction recoveries for the three index compounds were determined at low, medium, and high concentrations by comparing the peak areas of drugs in plasma samples with those from standard solutions at the same drug content levels.

Pharmacokinetic studies

\section{Animals and drug administration}

New Zealand white rabbits were selected for the study. The animals were fasted $24 \mathrm{~h}$ prior to the administration of drug formulations but had free access to water.

Twelve rabbits were divided equally into two groups between the male and female. The abdominal side hair of the rabbits was carefully shaved before transdermal administration. The $\mathrm{ME}$ gel and the $\mathrm{CP}$ were respectively placed on the skin of each rabbit for up to 24h. Blood samples were collected at preset time intervals of $0.5 \mathrm{~h} 、 1 \mathrm{~h} 、 1.5 \mathrm{~h} 、 2 \mathrm{~h} 、 4 \mathrm{~h} 、 6 \mathrm{~h} 、 8 \mathrm{~h} 、 10 \mathrm{~h}$. $12 \mathrm{~h} 、 24 \mathrm{~h}$ from the marginal ear vein of the rabbits after administration of the ME gel and CP.

\section{E. Blood Sample preparation}

The collected blood samples were centrifuged at $3000 \mathrm{r} / \mathrm{min}$ for $10 \mathrm{~min}$ to obtain the serum samples.
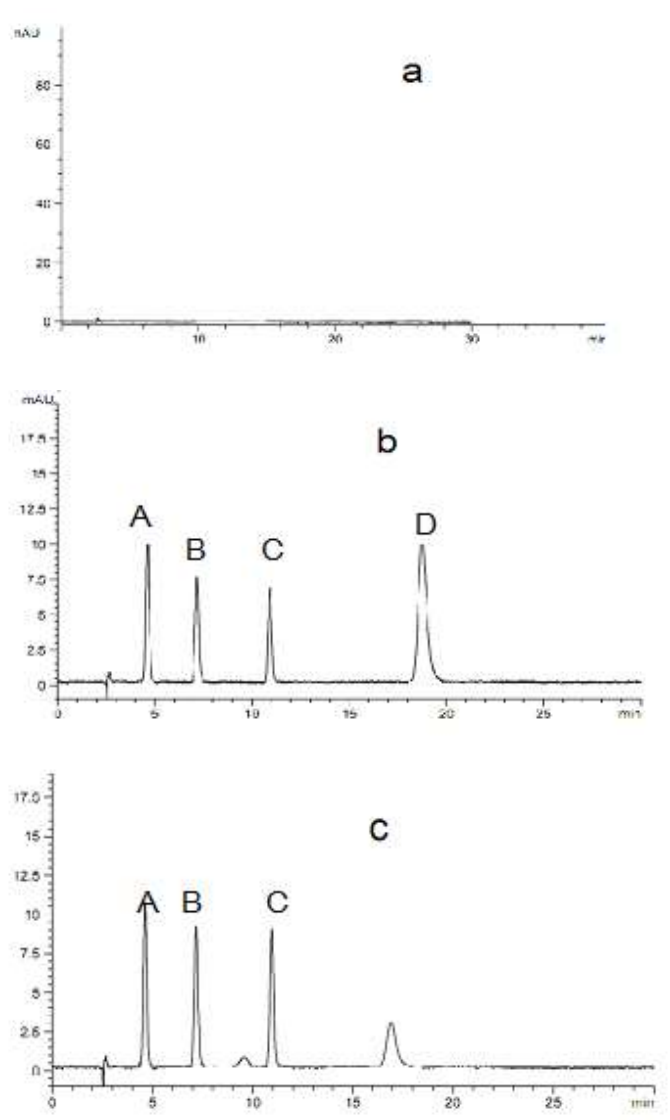

Figure 1. Chromatograms of blank plasma (a) and plasma spiked with the three index compounds of CCD and internal standard (b) and plasma spiked with CCD (c). Chromatographic peaks: (A) Daphnin, (B)

Daphnetin-8-O- $\beta$-D-glucoside, (C) Daphnetin, (D) ferulic acid (internal standard) 
Aliquots of $500 \mu \mathrm{L}$ serum plasma were pipetted into 5 $\mathrm{mL}$ centrifuge tubes and $40 \mu \mathrm{L}$ of ferulic acid were added. The mixture was extracted with $2 \mathrm{~mL}$ ethyl acetate, denatured with $1 \mathrm{~mL}$ methanol, vortexed for 2 min to ensure full extraction and deproteinization, followed by centrifugation for $2 \mathrm{~min}$ at $1000 \mathrm{r} / \mathrm{min}$. Both organic layers were transferred to a clean centrifuge tube and evaporated with air stream at $35^{\circ} \mathrm{C}$ to dry. The residue was reconstituted with $1 \mathrm{ml}$ of $50 \%$ methanol and filtered with $0.45 \mu \mathrm{m}$ microporous membrane to get the blood sample.

\section{RESULTS AND DISCUSSION}

\section{A. Validation of the HPLC method}

Retention times for the three compounds were found to be within $4.5 \sim 12 \mathrm{~min}$ and that for the internal standard was about $19 \mathrm{~min}$ with a total run time of 30 min. No endogenous interfering peaks were observed in the individual blank serum at the three compounds and ferulic acid retention times, thus confirming the analytical method's specificity. (Fig .1)

Standard plots for daphnin, daphnetin-8-O-

TABLE I PRECISION AND EXTRACTION RECOVERY OF THE THREE INDEX COMPOUNDS IN RABBIT PLASMA (N=3 DAYS, SIX REPLICATES PER DAY)

\begin{tabular}{|c|c|c|c|c|c|c|c|}
\hline \multirow{2}{*}{ compound } & \multirow{2}{*}{$\begin{array}{c}C \\
(\mu \mathrm{g} / \mathrm{mL})\end{array}$} & \multirow{2}{*}{$\begin{array}{l}\text { Intra-day } \\
\text { RSD (\%) }\end{array}$} & \multirow{2}{*}{$\begin{array}{l}\text { Inter-day } \\
\text { RSD (\%) }\end{array}$} & \multirow{2}{*}{$\begin{array}{c}\text { Accuracy } \\
(\%)\end{array}$} & \multicolumn{3}{|c|}{ Extraction recovery $(\%)$} \\
\hline & & & & & individual & mean & $\operatorname{RSD}(\%)$ \\
\hline \multirow[t]{3}{*}{ Daphnin } & 4.72 & $2.41 \%$ & $2.07 \%$ & & 78.80 & & \\
\hline & 28.51 & $1.31 \%$ & $1.28 \%$ & 100.50 & 77.20 & 77.90 & 1.04 \\
\hline & 89.17 & $0.89 \%$ & $1.27 \%$ & & 77.80 & & \\
\hline Daphnetin-8-O- $\beta-\mathrm{D}-\mathrm{g}$ & 4.54 & $2.62 \%$ & $2.65 \%$ & & 77.30 & & \\
\hline \multirow[t]{2}{*}{ lucoside } & 27.41 & $1.33 \%$ & $1.58 \%$ & 100.60 & 78.00 & 78.50 & 1.93 \\
\hline & 85.73 & $0.88 \%$ & $0.91 \%$ & & 80.20 & & \\
\hline \multirow[t]{3}{*}{ Daphnetin } & 2.99 & $3.27 \%$ & $3.15 \%$ & & 78.40 & & \\
\hline & 18.06 & $1.31 \%$ & $1.88 \%$ & 96.70 & 79.50 & 79.40 & 1.14 \\
\hline & 56.47 & $1.02 \%$ & $0.99 \%$ & & 80.20 & & \\
\hline
\end{tabular}

\section{B. Pharmacokinetic study}

The concentrations of the three components of CCD in plasma were determined and used to study the pharmacokinetic of the drug. The plasma concentration-time profiles of the three compounds are displayed in Fig .2.

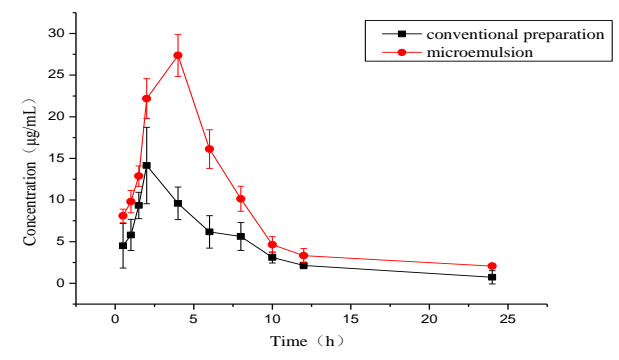

(a)
$\beta$-D-glucoside, and daphnetin in plasma were linear in the concentration range $1.82 \sim 90.90 \mu \mathrm{g} / \mathrm{mL} 、 1.75 \sim 87.70$ $\mu \mathrm{g} / \mathrm{mL}$, and $1.18 \sim 59.20 \mu \mathrm{g} / \mathrm{mL}$ respectively with correlation coefficient values of 0.9999 . The limits of quantitation (LOQ) of the method for the above mentioned samples were found to be $0.908 \mu \mathrm{g} / \mathrm{mL}$, $0.873 \mu \mathrm{g} / \mathrm{mL}$, and $0.576 \mu \mathrm{g} / \mathrm{mL}$ respectively in rabbit plasma. The limits of detection (LOD) were determined to be $8.17 \mathrm{ng} / \mathrm{mL}, 7.86 \mathrm{ng} / \mathrm{mL}$, and $5.76 \mathrm{ng} / \mathrm{mL}$ for the three compounds based on signal-to-noise ratio of 3:1 in rabbit plasma. The precisions of the QC samples were satisfactory with RDS less than $4 \%$. The average recovery values for daphnin, daphnetin-8-O- $\beta$-D-glucoside, and daphnetin were $100.50 \%, 100.60 \%$, and $96.70 \%$ respectively ensuring consistency and efficiency of the analysis method(Table $1)$.

The mean extraction recoveries for daphnin, daphnetin-8-O- $\beta$-D-glucoside, and daphnetin in low, medium, and high concentrations were found to be $77.90 \%, 78.50 \%$ and $79.40 \%$ respectively (Table 1 ). 


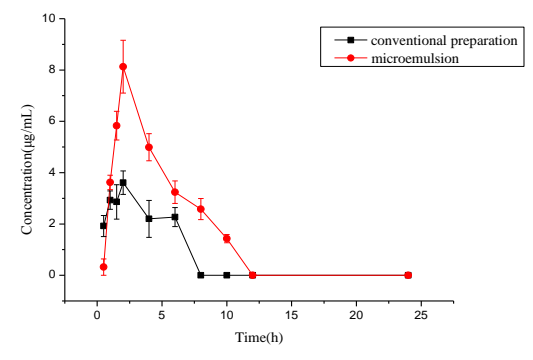

(c)

Figure 2. Mean plasma concentration-time profiles of daphnin(a), daphnetin-8-O- $\beta$-D- glucoside(b), and daphnetin(c) in rabbits.

The concentrations of daphnin, daphnetin-8-O- $\beta$-Dglucoside, and daphnetin in rabbit plasma increased rapidly after administration of $\mathrm{CP}$ and reached their maxima within $2 \mathrm{~h}$. When the ME gel was applied, the concentrations of the three compounds were a little higher than that of $\mathrm{CP}$ at each time interval. Higher Cmax values were observed for the compounds in $\mathrm{ME}$ gel than those of $\mathrm{CP}$, which suggested that a larger amount of CCD was absorbed and accumulated in the blood stream when administered with the ME gel.

Pharmacokinetic parameters of the three index compounds of CCD were estimated by using the DAS 2.1.1 program. Statistical moment analysis was used to calculate the pharmacokinetic parameters Cmax, Tmax , $\operatorname{AUC}(0-\infty)$, and $t 1 / 2$. (Table 2 )

The ME formulation acted as a delivery vehicle for the drug components, which allowed them to pass through the skin barrier successfully and reached equilibrium gradually in blood circulation with prolonged action time and improved efficacy. This is confirmed by the Cmax and AUC(0-t) values of the compounds in two formulation. The results showed a significant improvement of the AUC $(0-t)$ s for the three compounds, resulting in a totally 1.91 -fold increased relative bioavailability and approximately 2-fold improved Cmax as compared to that in CP. The enhanced and sustained uptake is likely caused by the combination of improved transport of the drug solubilized in small oil globules that easily transport through lipids of stratum corneum in the skin and the sustained release of the drug components from the ME gel.

TABLE II PHARMACOKINETIC PARAMETERS CALCULATED WITH NON COMPARTMENT ANALYSIS (MEAN \pm SD, N=6)

\begin{tabular}{|c|c|c|c|c|c|c|}
\hline \multirow{2}{*}{ Parameters } & \multicolumn{2}{|c|}{ daphnin } & \multicolumn{2}{|c|}{ daphnetin-8-O-p-D-glucoside } & \multicolumn{2}{|c|}{ daphnetin } \\
\hline & gel & $\mathbf{C P}$ & gel & $\mathbf{C P}$ & gel & $\mathbf{C P}$ \\
\hline $\begin{array}{c}\mathrm{AUC}_{(0-\mathrm{t})}\left(\mathrm{mg} \mathrm{g}^{*} \mathrm{~h}\right. \\
/ \mathrm{L})\end{array}$ & $200.03 \pm 61.73$ & $97.82 \pm 29.08$ & $158.79 \pm 54.43$ & $63.98 \pm 20.12$ & $41.02 \pm 9.16$ & $14.78 \pm 3.42$ \\
\hline $\begin{array}{l}\operatorname{AUC}_{(0-\infty)} \\
(m * h \mathrm{~g} / \mathrm{L})\end{array}$ & $212.64 \pm 65.09$ & $98.31 \pm 31.42$ & $162.13 \pm 49.65$ & $71.36 \pm 24.78$ & $47.39 \pm 16.03$ & $57.75 \pm 17.92$ \\
\hline $\mathrm{t}_{1 / 2 \mathrm{z}}(\mathrm{h})$ & $2.76 \pm 0.37$ & $4.07 \pm 0.65$ & $3.77 \pm 1.03$ & $5.92 \pm 0.93$ & $3.45 \pm 0.89$ & $13.11 \pm 4.15$ \\
\hline $\mathrm{T}_{\max }(\mathrm{h})$ & $4.00 \pm 0.41$ & $2.00 \pm 0.21$ & $4.00 \pm 0.38$ & $2.00 \pm 0.18$ & $2.00 \pm 0.19$ & $2.00 \pm 0.25$ \\
\hline $\begin{array}{c}\mathrm{C}_{\max } \\
(\mathrm{mg} / \mathrm{L})\end{array}$ & $28.62 \pm 8.74$ & $13.97 \pm 3.21$ & $15.87 \pm 4.02$ & $8.43 \pm 2.36$ & $8.09 \pm 2.13$ & $3.58 \pm 1.20$ \\
\hline $\begin{array}{c}\mathrm{CL}_{2} / \mathrm{F} \\
(\mathrm{L} / \mathrm{h} / \mathrm{kg})\end{array}$ & $0.13 \pm 0.01$ & $0.23 \pm 0.03$ & $0.13 \pm 0.02$ & $0.26 \pm 0.03$ & $0.45 \pm 0.08$ & $0.36 \pm 0.04$ \\
\hline $\begin{array}{l}\mathrm{V}_{\mathrm{z}} / \mathrm{F} \\
(\mathrm{L} / \mathrm{kg})\end{array}$ & $0.39 \pm 0.09$ & $1.15 \pm 0.33$ & $0.64 \pm 0.17$ & $2.35 \pm 0.58$ & $2.14 \pm 0.39$ & $6.65 \pm 1.82$ \\
\hline
\end{tabular}

\section{CONCLUSIONS}

A specific and sensitive HPLC method was developed for the simultaneous quantification of the three index components of coumarins from cortex daphnes in rabbit plasma and successfully applied to the pharmacokinetic study of the active compounds after transdermal delivery the ME gel of CCD. The ME gel formulation was efficacious for the delivery of CCD and showed no skin irritation for transdermal administration. Results of bioavailability study showed improved permeation of the drug components from the $\mathrm{ME}$ gel compared to $\mathrm{CP}$ formulation. These results suggest that the prepared system shows potential to sustain the transdermal delivery of CCD for the treatment of rheumatism and other similar diseases.

\section{REFERENCES}

[1]. Kang AL, Li W, Zhang X, Tang YS, "Experimental research of effective components in Daphnes folium extracted by percolation method", China Pharm, vol. 15, 2012, pp.1248-1250.

[2]. Wang P, Liu JP, Zhan N, Li YP, Lu D, "Progress in the research on chemical constituents and pharmacological activities of 
Zushima”, Spec Wild Econ Anim Plant Res, vol. 4, 2011. pp. 73-76.

[3]. LI K, Y RL,Kong Y,Zhang L,Zhu SY,Chen LQ, "Optimization of purification technology of total coumarins in Daphne giraldii", Chin J Exp Tradit Med Form, vol. 19, 2013, pp.58-60.

[4]. Zhao XH, He JT, Tu HH, Zhang JC, "Study on preparation of Zhitong spray", Chin New Drugs J, vol. 21, 2012, pp. 2562-2565

[5]. Kang Along, Li Wei, Sun Chengrong, Zhang xian, "Recent developments in the chemical constituents and pharmaceutical preparation of Zushima", Northwest Pharm J, vol. 26, 2011, pp. 479-482.

[6]. Li S, Shi XF, Fan B, Bai CH, "Determination of residual amounts of daphnetin and 7-hydroxycoumarin in Zushima Plaster used externally", Chin Tradit Pat Med, vol. 35, 2013, pp.1209-1212.
[7]. Duan H, Mu FX, "Clinical observation of Zhushima Tincture in the treatment of acute soft tissue contusion", Chin Tradit Pat Med, vol. 34,2012, pp.1447-1451.

[8]. Talegaonkar S, Azeem A, Ahmad FJ, "Microemulsions: a novel approach to enhanced drug delivery", Recent Pat Drug Deliv Formul, vol. 2, 2008, pp.238-257.

[9]. Lawrence MJ, Rees GD, "Microemulsion-based media as novel drug delivery systems", Adv Drug Deliv Rev, vol. 45, 2000, pp.89-121.

[10]. Kogan A, Garti N, "Microemulsions as transdermal drug delivery vehicles", Adv Colloid Interface Sci., vol. 123,2006, pp. 369-385. 